\title{
PRODUCTION AND STUDY OF NEUTRON-RICH NUCLEI USING THE LICORNE DIRECTIONAL NEUTRON SOURCE*
}

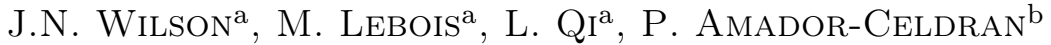

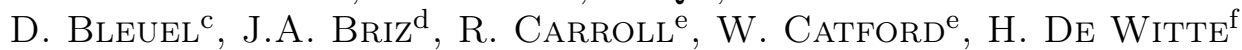

D. Doherty ${ }^{\mathrm{g}}$, R. Eloirdi ${ }^{\mathrm{b}}$, G. Georgiev ${ }^{\mathrm{h}}$, A. Gottardo $^{\mathrm{a}}$

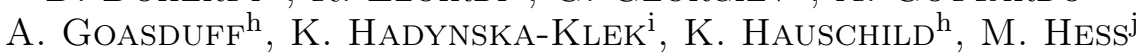

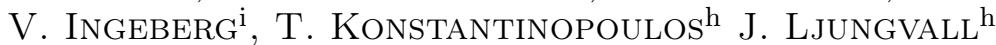

A. Lopez-Martens ${ }^{h}$, G. Lorusso ${ }^{k}$, R. Lozeva ${ }^{\text {l,h }}$, R. Lutter ${ }^{\mathrm{m}}$

P. Marini ${ }^{\mathrm{n}}$, I. Matea ${ }^{\mathrm{a}}$, T. Materna ${ }^{\mathrm{g}}$, L. Mathied $^{\mathrm{n}}$, A. Oberstedt $^{\mathrm{o}}$

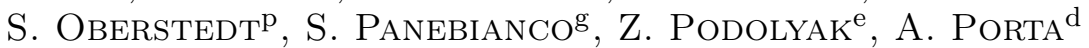

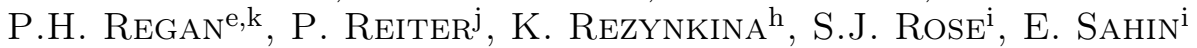

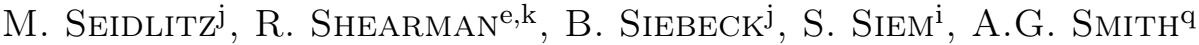

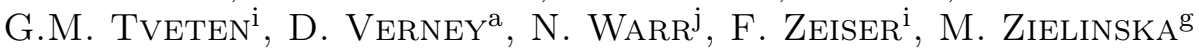

${ }^{a}$ Institut de Physique Nucleaire d'Orsay

Bat. 100, 15 rue G. Clemenceau, 91406 Orsay cedex, France

${ }^{\mathrm{b}}$ European Commission, Joint Research Centre

Directorate G for Nuclear Safety and Security

Unit G.I.5, Postfach 2340, 76215 Karlsruhe, Germany

${ }^{\mathrm{c}}$ Lawrence Livermore National Laboratory, 7000 East Ave, Livermore, CA 94550, USA

${ }^{\mathrm{d}}$ Subatech/Ecole des Mines, 4 rue Alfred Kastler, 44307 Nantes, France

${ }^{\mathrm{e}}$ Department of Physics, University of Surrey, Guildford, GU2 7XH, UK

${ }^{\mathrm{f}} \mathrm{KU}$ Leuven, 3000 Leuven, Belgium

${ }^{g}$ Irfu, CEA, Université Paris Saclay, 91191 Gif-sur-Yvette Cedex, France

${ }^{\mathrm{h}}$ CSNSM Orsay, Bat. 104, 91405 Orsay, France

${ }^{\mathrm{i}}$ University of Oslo, Department of Physics, P.O. Box 1048, Blindern 0316 Oslo, Norway

${ }^{\mathrm{j}}$ Institut fur Kernphysik, Zülpicher Strasse 77, 50937 Köln, Germany

${ }^{\mathrm{k}}$ National Physical Laboratory, Hampton Road, Teddington, Middlesex TW11 0LW, UK

${ }^{1}$ IPHC, Université de Strasbourg, 23 rue du Loess, BP28 67037, Strasbourg, France

${ }^{\mathrm{m}}$ LMU München, Schellingstrasse 4, 80799, Munich, Germany

${ }^{\mathrm{n}}$ CENBG Bordeaux, Chemin du Solarium, Le Haut Vigneau BP 12033175 Gradignan, France

${ }^{\circ}$ Extreme Light Infrastructure-Nuclear Physics (ELI-NP)

077125 Bucharest-Măgurele, Romania

${ }^{\mathrm{p}}$ European Commission, Joint Research Centre

Directorate for Nuclear Safety and Security

Unit G.2, 2440 Geel, Belgium

${ }^{\mathrm{q}}$ University of Manchster, Dept. of Physics, Oxford Road, Manchester, M13 9PL, UK

(Received January 19, 2017)

* Presented at the Zakopane Conference on Nuclear Physics "Extremes of the Nuclear Landscape", Zakopane, Poland, August 28-September 4, 2016. 
We have recently successfully demonstrated a new technique for production and study of many of the most exotic neutron-rich nuclei at moderate spins. LICORNE, a newly developed directional inverse-kinematic fast neutron source at the IPN Orsay, was coupled to the MINIBALL high resolution $\gamma$-ray spectrometer to study nuclei the furthest from stability using the ${ }^{238} \mathrm{U}(n, f)$ reaction. This reaction and ${ }^{232} \mathrm{Th}(n, f)$ are the most neutron-rich fission production mechanisms achievable and can be used to simultaneously populate hundreds of neutron-rich nuclei up to spins of $\approx 16 \hbar$. High selectivity in the experiment was achieved via triple $\gamma$-ray coincidences and the use of a 400 ns period pulsed neutron beam, a technique which is unavailable to other population mechanisms such as ${ }^{235} \mathrm{U}\left(n_{\mathrm{th}}, f\right)$ and ${ }^{252} \mathrm{Cf}(\mathrm{SF})$. The pulsing allows time correlations to be exploited to separate delayed $\gamma$ rays from isomeric states in the hundreds of nuclei produced, which are then used to cleanly select a particular nucleus and its exotic binary partners. In the recent experiment, several physics cases are simultaneously addressed such as shape coexistence, the evolution of shell closures far from stability, and the spectroscopy of nuclei in the r-process path near $N=82$. Preliminary physics results on anomalies in the ${ }^{238} \mathrm{U}(n, f)$ fission yields and the structure of the ${ }^{138} \mathrm{Te}$ and ${ }^{100} \mathrm{Sr}$ nuclei will soon be published. A future project, $\nu$-ball, to couple LICORNE with a hybrid escape-suppressed spectrometer to refine further the technique and achieve a large increase in the observational limit is discussed.

DOI:10.5506/APhysPolB.48.395

\section{Introduction}

Over 7000 bound nuclei are theoretically predicted to exist, yet only around 4000 of these are currently accessible to study in the laboratory. This is due to the limited production mechanisms available here on earth. The problem is particularly difficult on the neutron-rich side of the nuclear chart, where for nuclei above $Z>30$, the neutron drip-line is predicted far beyond what is currently known. At the edge of current knowledge, typically only one or two excited nuclear states are known at best. One production mechanism often used to access the most exotic neutron-rich nuclei is nuclear fission. However, not all fission reactions are the equivalent. Figure 1 compares various fission reactions used to populate neutron-rich nuclei.

Gamma-ray spectroscopy has proved to be a useful tool to study the fission fragments in nuclear fission in the past and, indeed, a wealth of information has been obtained about neutron-rich fission fragments in the 1990s and 2000s with the Gammasphere and Euroball arrays studying spontaneous fission sources of ${ }^{252} \mathrm{Cf}$ and ${ }^{248} \mathrm{Cm}[1,2]$. Studies of excited states at medium spins (typically 16 $\hbar$ ) are accessible in these types of experiments, and are complementary to the $\beta$-decay studies at radioactive beam facilities, which only probe states of very low spin. More recently, the EXILL experimental 
campaign [3, 4], which used the Exogam array at the ILL reactor, performed $\gamma$-ray spectroscopy of the thermal neutron induced fission of ${ }^{235} \mathrm{U}$ and ${ }^{241} \mathrm{Pu}$ reactions. By changing the fissioning system, the fission yields change giving access to different sets of isotopes. However, to reach the most exotic neutron-rich nuclei requires the use of the ${ }^{232} \mathrm{Th}(n, f)$ and ${ }^{238} \mathrm{U}(n, f)$ reactions (see Fig. 1). These reactions are difficult to study since they require an intense, directional source of fast neutrons coupled to a high-resolving power Germanium spectrometer, yet an early test showed a potential [5]. In this work, we report on the recent successful demonstration of a technique to study exotic nuclei populated in the ${ }^{238} \mathrm{U}(n, f)$ reaction. This experiment used the LICORNE high flux, naturally directional neutron source developed at the IPN Orsay coupled to the MINIBALL spectrometer [6].

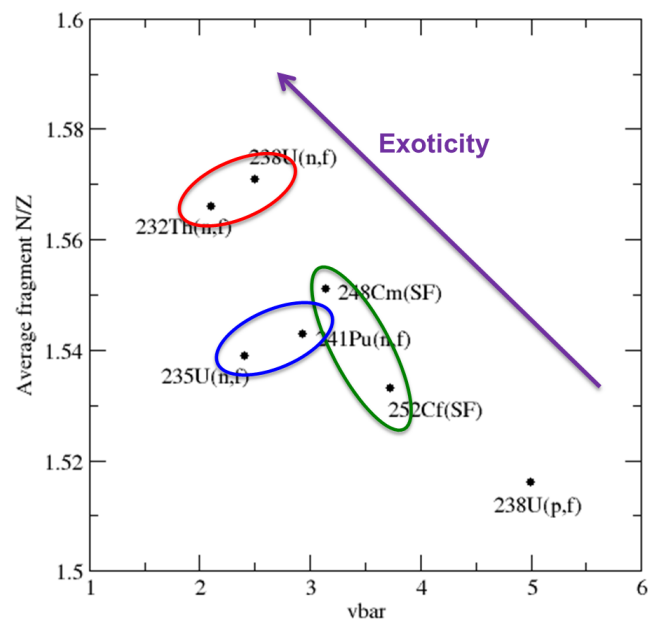

Fig. 1. (Colour on-line) Comparison of various fission reactions with the exoticity $(N / Z$ ratio) of the fragments after neutron emission plotted as a function of the average neutron multiplicity $\nu$-bar taken from ENDFB.VII. The spontaneous fission sources are circled in light grey/green. The thermal neutron induced fission reactions used in the EXILL campaign are circled in black/blue. The reactions that need fast neutrons are circled in grey/red.

\section{Production of directional neutrons in inverse kinematics}

The LICORNE neutron source $[7,8]$ produces fast directional neutrons using the inverse reaction $p\left({ }^{7} \mathrm{Li}, n\right){ }^{7} \mathrm{Be}$. Intense ${ }^{7} \mathrm{Li}$ beams provided by the Tandem accelerator of the ALTO facility at the IPN Orsay impinge on a gaseous hydrogen target. The gas cells operate between 1 and 2 atmospheres pressure and have lengths of between 2 and $7.5 \mathrm{~cm}$ to provide a complete 
control of the number of hydrogen atoms in the beam. Neutrons are emitted in cones of up to 25 degrees opening angle producing neutrons in the 0.5 to $4 \mathrm{MeV}$ range.

The goal has been to couple LICORNE to a Germanium spectrometer with a high resolving power in order to select and study particular nuclei with double $\gamma-\gamma$ coincidences or triple $\gamma-\gamma-\gamma$ coincidences. To perform such a coupling, it was first necessary to eliminate all secondary, parasitic neutron fields generated by unwanted nuclear reactions, since Germanium detectors are very sensitive to neutron damage. Practically, this meant optimising the design to use high- $Z$ materials (tungsten and lead) for collimation and stopping of the ${ }^{7} \mathrm{Li}$ beam, and using tantalum as the material for the thin entrance window. This window, which separates the beam line vacuum from the pressurised and circulating hydrogen gas, is ultra thin $(2-3 \mu \mathrm{m})$ and has to withstand extreme conditions of temperature, pressure differential and irradiation damage.

In February 2015, LICORNE was successfully coupled to the MINIBALL spectrometer at the IPN Orsay, a spectrometer which was on loan from the MINIBALL Collaboration and based at the ISOLDE facility at CERN. MINIBALL consists of 24 Ge crystals packaged in 8 triple-clusters (see Fig. 2). Each crystal was approximately $14 \mathrm{~cm}$ from the fission target, and the array covered around $33 \%$ of the full solid angle providing a total photopeak efficiency of around $6 \%$ at $1 \mathrm{MeV}$.

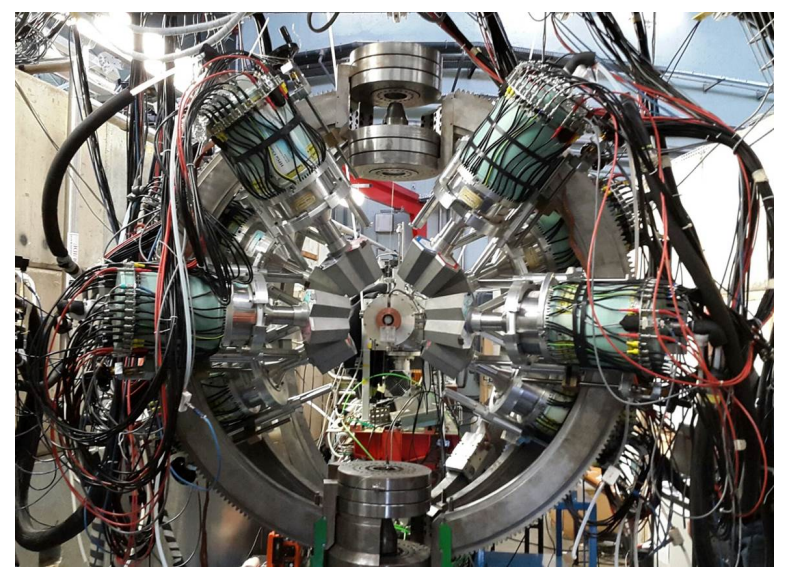

Fig. 2. Photograph of the successful coupling of the LICORNE neutron source and the MINIBALL array.

The gas cell used was $3.5 \mathrm{~cm}$ in length at a pressure of 1.5 atmospheres and a tantalum window of thickness of $2.7 \mu \mathrm{m}$. The ${ }^{7} \mathrm{Li}$ bombarding energy was chosen to be $16.75 \mathrm{MeV}$, to allow the beam to exit the tantalum window at $\approx 15 \mathrm{MeV}$, then losing sufficient energy in the cell to reach the reaction 
threshold at $13.1 \mathrm{MeV}$ at the beam stop, thus maximizing neutron flux. The neutrons produced had an average energy of $1.7 \mathrm{MeV}$ just above the ${ }^{238} \mathrm{U}$ fission threshold at $1.2 \mathrm{MeV}$. The maximum cone angle was 19.5 degrees and the neutrons were constrained by the kinematics within a distribution between $700 \mathrm{keV}$ and $3 \mathrm{MeV}$. The beam was pulsed with a period of $400 \mathrm{~ns}$ which not only allowed direct access to time correlations, but also helped to separate fission events from uncorrelated $\beta$-decay background. This is a key advantage to the experimental technique that is unavailable for spontaneous fission and thermal neutron induced fission.

A depleted uranium metal half-cylinder $\left(99.78 \%{ }^{238} \mathrm{U}\right)$ of length $2.98 \mathrm{~cm}$, radius $0.57 \mathrm{~cm}$ and a mass of $31.35 \mathrm{~g}$ was used as the sample and placed along the beam axis at a distance of $3 \mathrm{~cm}$ from the end of the gas cell. The average flux seen by the sample was $\approx 2 \times 10^{6} \mathrm{n} / \mathrm{cm}^{2} / \mathrm{s}$ inducing a fission rate estimated from Geant 4 simulations as $25 \mathrm{kHz} .2 .8 \times 10^{9} M_{\gamma} \geq 3$ coincidence events were collected in around 11 days of effective beam time. Data were collected at the maximum rate achievable, which was limited by a maximum $\approx 4 \mathrm{kHz}$ MINIBALL trigger rate and an $\approx 8 \mathrm{kHz}$ Ge singles rate. The pulsed ${ }^{7} \mathrm{Li}$ beam current was $32 \mathrm{nA}$ and was not the limiting factor in the experiment.

The MINIBALL array was designed as a high-efficiency, low-multiplicity device for radioactive beam experiments and is not Compton suppressed. The peak-to-total is fairly low, $22 \%$, and thus limits the resolving power of the device when used to select nuclei using standard double or triple $\gamma$-coincidence techniques. The Compton background in MINIBALL is particularly large at low energies of $200-500 \mathrm{keV}$ and limits the sensitivity of the experiment in this energy range. Special analysis techniques had to be developed to deal with events in which scattering of $\gamma$ rays between two detectors in MINIBALL sums to the incident full photopeak energy, which produces diagonal lines/planes in $2 \mathrm{D} / 3 \mathrm{D}$ histograms. It is essential that these counts are subtracted to prevent the appearance of false coincidences when gating.

Another experimental difficulty was the geometry of the uranium metal target and the high density of uranium $\left(19.1 \mathrm{~g} / \mathrm{cm}^{3}\right)$ which attenuates lowenergy $\gamma$ rays strongly. Future improvements to the technique will include arranging a similar mass of uranium metal in either a stack of thin discs, or as an encapsulated metal or oxide powder, to decrease both average density and the consequent attenuation of the low-energy $\gamma$ rays from fission events within the body of the sample.

\section{Spectroscopy of ${ }^{238} \mathrm{U}(n, f)$ near the reaction threshold}

Figure 3 shows a typical $\gamma$-ray spectrum produced when gating on a single strong transition, in this case the $2^{+}$to $0^{+}$transition in ${ }^{96} \mathrm{Sr}$ which is 
measured to be produced at $2.7 \%$ of the total fission yield in this reaction. As expected, other lines from ${ }^{96} \mathrm{Sr}$ appear in coincidence, along with transitions from the binary partners ${ }^{139,140,141,142} \mathrm{Xe}$ corresponding to $4 n, 3 n, 2 n$ and $1 n$ channels, respectively.

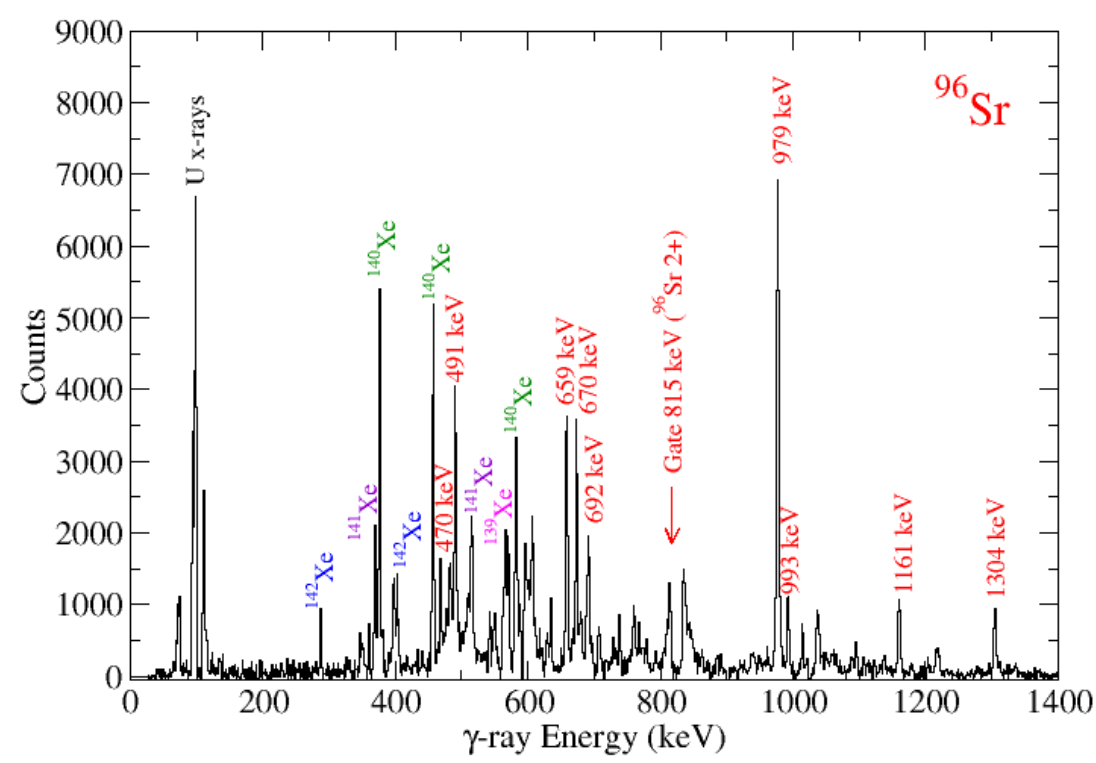

Fig. 3. Spectrum of $\gamma$ rays in coincidence with the $815 \mathrm{keV}$ transition in ${ }^{96} \mathrm{Sr}$.

Preliminary analysis of the data shows that we comfortably populate certain nuclei which are difficult to populate in spontaneous fission. The centroids and tails of the yield distributions for a given isotope typically shift up to two nucleons more to the right on the nuclear chart. In addition, the observational limit is improved over spontaneous fission since fewer fission products are produced, the fragments confined to narrower ranges in $Z$, and the coupling between binary partners is much stronger since the average neutron multiplicity is lower (2.5 compared to 4).

For example, the ${ }^{138} \mathrm{Te}$ isotope has recently been studied at Riken in $\beta$ decay [9], which we populate easily in our experiment at medium spins. Extrapolating our measured tellurium yields suggests that ${ }^{139} \mathrm{Te}$ and ${ }^{140} \mathrm{Te}$ are within the observational limit. For ${ }^{140} \mathrm{Te}$, any new $\gamma$ rays will have to be identified from its binary partners.

Data analysis is ongoing, but preliminary results from this experiment suggest, for example, new spectroscopic information for the ${ }^{138} \mathrm{Te}$ and ${ }^{100} \mathrm{Sr}$ nuclei. Furthermore, large discrepancies in the charge yields for certain isotopes have been observed with respect to current fission-yield evaluations for this incident neutron energy. 
The pulsed beam has also allowed gating on delayed isomeric transitions with half-lives of around $20-2000 \mathrm{~ns}$ (e.g. the $167 \mathrm{~ns}$ isomer in ${ }^{134} \mathrm{Te}$ [10] is populated strongly). The idea is to use the exceptional selectivity of the time correlations to pick out fragments with isomers close to stability and their corresponding binary partners which will be far from stability. This idea, which we call TIPS (Tagging Isomer PartnerS), has yet to realise its full potential due to the break-through of an uncorrelated background from $\beta$ decay amongst the delayed events. This can only be reduced by a more accurate triggering upon genuine fission events. In the future, we plan to refine the technique by coupling the LICORNE neutron source to the $\nu$-ball escape-suppressed spectrometer which will open up new possibilities for the precision spectroscopy of neutron induced reactions. $\nu$-ball will be a hybrid, escape suppressed $\gamma$-ray spectrometer coupling both $\mathrm{Ge}$ and $\mathrm{LaBr}_{3}$ detectors to approach around $10 \%$ photopeak efficiency. The escape suppression is expected to increase the resolving power by at least a factor of ten, and allow access to sub-nanosecond time ranges. Furthermore, sub-nanosecond timing and calorimetry will be used to gain orders of magnitude improvement in the separation of $\beta$ decay and fission events, and thus increase the selectivity of the isomer tagging. In conclusion, LICORNE and $\nu$-ball may open up interesting new possibilities in the spectroscopy of neutron-rich isotopes and the population and study of fission isomers.

We would like to thank the staff at the ALTO facility for the production of high intensity ${ }^{7} \mathrm{Li}$ beams and the German BMBF under contract 05P15PKCIA (ISOLDE) and Verbundprojekt 05P2015.

\section{REFERENCES}

[1] J.K. Hwang et al., Phys. Rev. C 57, 2250 (1998).

[2] A.G. Smith et al., Phys. Rev. C 60, 064611 (1999).

[3] G. de France et al., Eur. Phys. J. Web Conf. 66, 02010 (2014).

[4] P. Baczyk et al., Phys. Rev. C 91, 047302 (2015).

[5] W.R. Phillips et al., Eur. Phys. J. A 3, 205 (1998).

[6] N. Warr et al., Eur. Phys. J. A 49, 40 (2013).

[7] M. Lebois et al., Nucl. Instrum. Methods Phys. Res. A 735, 145 (2014).

[8] J.N. Wilson et al., Phys. Proc. 64, 107 (2015).

[9] P. Lee et al., Phys. Rev. C 92, 044320 (2015).

[10] R.E. Sund, H. Weber, V.V. Verbinski, Phys. Rev. C 10, 853 (1974). 